\title{
Past 4 Weeks
}

National Cancer Institute

\section{Source}

National Cancer Institute. Past 4 Weeks. NCI Thesaurus. Code C110986.

A period of time consisting of the 28 days before the present. 